\title{
Structure, forme et substance dans la théorisation martinettienne de la langue : la problématique des rapports formelsubstance comme obstacle épistémologique
}

\author{
Anne-Gaëlle Toutain \\ Laboratoire d'Histoire des Théories Linguistiques (HTL) - UMR 7597 CNRS - Université Paris \\ Sorbonne (France) \\ Institut de langue et de littérature françaises - Université de Berne (Suisse) \\ annegaelletoutain@yahoo.fr
}

\begin{abstract}
Résumé. Cet article s'attache à l'examen de la distinction martinettienne entre forme et substance, afin d'en faire apparaître la nature d'obstacle épistémologique. Après un rapide examen de la critique martinettienne de Hjelmslev sur cette question des rapports entre forme et substance, qui permet d'opposer problématiques saussurienne -théorique, définitoire de la langue comme un fonctionnement dont son et sens, en tant que linguistiques, sont les effets et structuraliste - problématique empirique de construction de la langue comme un existant formel -, il s'efforce ainsi de montrer le caractère contradictoire du fonctionnement de cette distinction dans l'élaboration martinettienne. Cette distinction détermine la construction d'une structure tout à la fois formelle et substantielle, et en réalité doublement déterminée, par la problématique des rapports forme/substance et par celle des rapports son/sens. La problématique des rapports son/sens détermine pour sa part la construction d'une structure orientée du son vers le sens, dont l'examen révèle l'asymétrie, asymétrie qui nous reconduit à la dualité de détermination de la construction martinettienne de la langue comme structure, et qui témoigne de l'insistance du problème de la théorisation du rapport son/sens. A cet égard, l'élaboration martinettienne apparaît tout à la fois radicalement différente de celle de Saussure, et significativement différente de celle de Hjelmslev, dont elle met en valeur le caractère de pendant objectal de la théorisation saussurienne de la langue, ainsi que d'autres élaborations structuralistes. Elle est ainsi tout particulièrement propre à faire apparaître la nécessité d'une réflexion sur la problématique de la linguistique contemporaine.
\end{abstract}

\begin{abstract}
This article studies the Martinettian distinction between form and substance, in order to show how it can be seen as an obstacle épistémologique (Bachelard). It analyzes first the Martinettian criticism of Hjelmslev's theory, concerning this question of the relationship between form and substance. Saussurean and structuralist problematics are thus contrasted: the first is theoretical and defines language (la langue) as a functioning of which sound and meaning, qua linguistic, are effects; the latter is an empirical problematics which constructs language as a formal object. We thus bring to light the contradictory functioning of the form/substance distinction in Martinettian thought: this leads to the construction of a structure that is at the same time formal and substantial, and which is in fact determined equally by the problematics of the form/substance relationships, and by that of the sound/meaning relationships. As for the problematics of the sound/meaning relationships, this leads to the construction of a sound to meaning oriented structure, whose study reveals an asymmetry.
\end{abstract}


This asymmetry is again symptomatic of the twofold determination of the Martinettian structure, and demonstrates the need of a theorization of the sound/meaning relationship. From this point of view, Martinettian theory appears both radically different to the Saussurean approach, and significantly distinct from Hjelmslevian thought, whose nature of objectal counterpart of the Saussurean theorization of language is thus brought to light, and from others structuralists's elaborations. It is consequently appropriate to highlight the necessity to think about the problematics of contemporary linguistics.

\section{Introduction}

Il importe de faire apparaître la fondamentale différence de problématique qui sépare le structuralisme linguistique, au moins européen, de la théorisation saussurienne de la langue, dont ce dernier s'était voulu une mise en œuvre, celle-ci dût-elle impliquer remaniements et ruptures. Une telle relecture de l'histoire de la linguistique nous paraît en effet féconde à deux égards. D'une part, elle se fonde sur une lecture radicalement nouvelle de l'œuvre de Saussure, qui insiste sur la rupture constitutive de celle-ci : la distinction entre langue et idiome, dans le cadre de la théorisation du rapport son/sens, qui construit l’idiome comme résultat, au lieu de le considérer comme un donné indépassable. D’autre part, elle s'attache à mettre au jour les enjeux des élaborations structuralistes en tant qu'élaborations paradoxalement présaussuriennes, élaborations qui, en dépit du relatif désintérêt dans lequel sont tombés ces écrits, et du développement d'une riche et prolixe linguistique post-structuraliste, demeurent néanmoins influentes aujourd'hui. C’est ce deuxième aspect que nous voudrions privilégier dans cet article, qui sera ainsi consacré à l'examen d'une élaboration structuraliste en particulier, dont il s'agira de faire apparaître, au-delà de la différence de problématique avec Saussure, commune à l'ensemble du structuralisme européen, la spécificité, en tant que modalité révélatrice de la nature et des enjeux du structuralisme européen. À cet égard, la théorie du linguiste français André Martinet nous paraît un objet de choix, car propre à faire apparaître la nature d’obstacle épistémologique ${ }^{1}$ de la problématique des rapports forme/substance qui caractérise le structuralisme européen et qui continue d'être sous-jacente à nombre d'études linguistiques contemporaines. Cette problématique y fonctionne en effet de manière tout à la fois permanente et contradictoire, ce qui dénonce son caractère d’obstacle épistémologique. C’est ce que, après un rapide examen de l'opposition martinettienne à Hjelmslev sur cette question de la distinction forme/substance, nous nous efforcerons de montrer, en envisageant successivement les rapports établis par Martinet entre forme et structure, et la construction martinettienne de la langue comme structure orientée du son vers le sens.

\section{Hjelmslev, Martinet, Saussure : la problématique des rapports forme/substance}

Avec la double articulation - opposée à l'isomorphisme des deux plans -, la distinction forme/substance constitue un important point d’opposition entre Hjelmslev et Martinet. On lit ainsi dès le résumé précédant le célèbre compte rendu des Prolégomènes à une théorie du langage - parus en danois en 1943, sous le titre Omkring sprogteoriens grundlaggelse - que Martinet publia en 1946 dans le tome XLIII du Bulletin de la Société de linguistique de Paris ${ }^{2}$ : « [...] on se demande s'il est possible de suivre l'auteur lorsqu'il propose de faire complètement abstraction de la substance, phonique et sémantique » (Martinet, 2000 : p. 71). Il faut noter, dans cette courte citation, l'expression : " faire abstraction de la substance », qui témoigne que Martinet se situe dans la même problématique que Hjelmslev. Faire abstraction de la substance implique en effet de considérer celle-ci comme un champ d'objets à prendre - ou non - en compte, et, dès lors, à postuler l'existence de quelque chose de tel qu'une forme. Il sera de même question dans le corps du texte de "degré d'utilisation de la substance » (Martinet, 2000 : p. 94) et, dans « Structural Linguistics » (1953), de " maniement de ce que Hjelmslev appelle la "substance” » (Martinet, 1953 : p. 582 [nous traduisons]). Dans ce dernier texte, la distinction forme/substance se profile d'ailleurs de manière très nette. On y lit en effet : "La nature structurale du langage résulte du fait que chaque langue organise ces deux substances selon ses propres structures. » (Martinet, 1953: p. 582 [nous traduisons]), puis : « Ce que Hjelmslev appelle la “forme” est le type d'organisation auquel la substance, 
largement identique, est soumise dans toute langue. » (Martinet, 1953 : p. 583 [nous traduisons]), et enfin : « Le point de vue de Prague [dont Martinet se réclame] est clairement définissable dans le cadre de l'opposition hjelmslevienne entre forme et substance. » (Martinet, 1953 : p. 583 [nous traduisons]). La critique martinettienne de $\mathrm{Hjelmslev}^{3}$ apparaît ainsi avant tout révélatrice de l'existence d'un terrain commun au structuralisme européen. Or, ce terrain commun est en réalité symptomatique ou définitoire de la spécificité de la problématique structuraliste en regard de la problématique saussurienne ${ }^{4}$. En effet, il n'existe chez Saussure rien de tel qu'une distinction entre forme et substance. Les deux propositions du Cours de linguistique générale convoquées par Hjelmslev - « La linguistique travaille donc sur le terrain limitrophe où les éléments des deux ordres se combinent; cette combinaison produit une forme, non une substance. " (Saussure, 1972 : p. 157), "Autrement dit, la langue est une forme et non une substance (voir p. 157). » (Saussure, 1972 : p. 169) - appartiennent en effet au chapitre " La valeur linguistique », dont le premier paragraphe, où figure la première de ces deux propositions - à laquelle se réfère la seconde -, intitulé « La langue comme pensée organisée dans la matière phonique », s’attache à définir la langue, non comme une entité dotée d'une extériorité objectale - et par exemple comme un existant formel, forme hjelmslevienne ou structure martinettienne -, mais comme un fonctionnement, dont son et sens, en tant que linguistiques, sont les effets. Dans ce cadre, à la différence de ce qui a lieu dans les textes structuralistes, pour lesquels il faut donc parler, à la lumière de la théorisation saussurienne de la langue, de "problématique des rapports forme/substance ", les deux termes de la distinction forme/substance ne se situent pas du tout sur le même plan : substance désigne tout d'abord un élément entrant en jeu dans le fonctionnement qu'est la langue (celle-ci est en effet « articulation de la pensée dans la matière phonique ", pour reprendre le titre des éditeurs, qui résume parfaitement la définition saussurienne de la langue, donc combinaison de deux «masses amorphes », que l'on peut qualifier de «substances »: la pensée et la matière phonique); il est ensuite un qualificatif que Saussure refuse d'appliquer à la langue, refus qui le conduit à parler de forme; le terme de forme renvoie ainsi, non, comme celui de substance - ce serait contradictoire avec la définition de la langue comme fonctionnement -, à un existant mais, d'une part, à ce caractère « non substantiel », au sens de non objectal, de la langue, et d'autre part, comme il est très net dans la première formulation du Cours de linguistique générale - la seule qui apparaisse dans les notes d'étudiants, ainsi qu'on le verra dans les citations qui suivent -, au « produit » du fonctionnement qu'est la langue, à savoir l'idiome, de caractère effectivement structural. Le refus du qualificatif de substance est redondant dans les textes de Saussure. On lit tout d'abord dans les notes de Riedlinger (notes du deuxième cours) qui sont l'une des deux sources de ce paragraphe du Cours de linguistique générale :

\footnotetext{
«[...] (comparaison de deux masses amorphes: l'eau et l'air. Si la pression atmosphérique change, la surface de l'eau se décompose en une succession d'unités : la vague <= chaîne intermédiaire qui ne forme pas substance ! Cette ondulation représente l'union et pour ainsi dire l'accouplement de la pensée avec cette chaîne phonique qui est en elle-même amorphe. Leur combinaison produit une forme.>) » (Saussure, 1997 : p. 21-22).
}

Engler signale par ailleurs dans son édition critique du Cours de linguistique générale deux passages des notes de Saussure, tout aussi significatifs :

« <La nécessité qu’il y a de faire porter le principal effort de la théorie du langage sur nos premières distinctions peut se mesurer à ceci : Il ne s'écrit pour ainsi dire pas une ligne où les faits de langage ne soient représentés comme une matière. [biffé]> " (Saussure, 1967 : p. 276)

«Comme le langage n’offre sous aucune de ses manifestations une substance, mais seulement des actions combinées ou isolées de forces physiologiques, physiques, mentales ; - et comme néanmoins toutes nos distinctions, toute notre terminologie, toutes nos façons de parler sont moulées sur cette supposition involontaire d'une substance, on ne peut se refuser, avant tout, à reconnaître que la théorie du langage aura pour plus essentielle tâche de démêler ce qu'il en est de nos distinctions premières. » (Saussure, 2002 : p. 197 [voir Saussure, 1967 : p. 276]). 
On pourrait enfin citer toutes les propositions relatives aux fondamentales négativité et relativité (à un point de vue) des entités linguistiques, et qui sont partie prenante de l'élaboration du concept de valeur, présidant à la définition de la langue comme fonctionnement. La dimension de la " production » - « Leur combinaison produit une forme. » (nous soulignons) -, c'est-à-dire de l'effet de langue, donc du caractère étiologique de la définition saussurienne de la langue, qui rend compte de l'existence des idiomes, est quant à elle tout à fait lisible dans ce même passage des notes de Riedlinger du deuxième cours ${ }^{5}$, où elle s'articule de manière très claire à un refus de la définition de la langue comme existant objectal - le langage n'est pas un moule :

\begin{abstract}
« [...] le rôle <caractéristique> du langage vis-à-vis de la pensée ce n’est pas < d'être> un moyen phonique, matériel mais c’est de créer un milieu intermédiaire de telle $<$ nature $>$ que le compromis entre la pensée et le son aboutit d'une façon inévitable à des unités <particulières.> La pensée de sa nature chaotique est forcée de se préciser parce qu'elle <est> décomposée, elle est répartie par le langage en des unités. Mais il ne faut pas tomber dans l'idée banale que le langage est un moule : c'est le considérer comme quelque chose de fixe, de rigide alors que la <matière phonique est aussi> chaotique en soi que la pensée. <Ce n'est pas du tout cela: ce n'est pas la matérialisation de ces pensées par un son qui est un phénomène utile,> c'est le fait $<$ en quelque sorte> mystérieux que la pensée-son implique des divisions qui sont les unités finales de la linguistique. » (Saussure, 1997 : p. 21).
\end{abstract}

Il faut en effet noter dans ce passage, en lien avec la définition de la langue comme articulation ${ }^{6}$ de la pensée et du son, ces deux énoncés : «le compromis entre la pensée et le son aboutit d'une façon inévitable à des unités particulières », "c’est le fait en quelque sorte mystérieux que la pensée-son implique des divisions qui sont les unités finales de la linguistique » (nous soulignons).

Les notions saussuriennes de forme et de substance apparaissent ainsi significativement différentes de leurs homonymes structuralistes, qui renvoient pour leur part à deux types d'existants, dont l'un, la forme, est définitoire de la langue. Là où Saussure théorise l'idiome dans le cadre de la construction du concept de langue, les structuralistes définissent d'emblée la langue comme forme, définition qui se révèle ainsi, à la lumière récurrente de la théorisation saussurienne de la langue, sans rupture avec la connaissance commune. Aussi, précisément, la distinction forme/substance fait-elle figure d’obstacle épistémologique, comme il apparaît de manière d'autant plus nette à l'analyse des textes de Martinet que, dans l'élaboration de ce dernier, le primat de la problématique des rapports son/sens fait obstacle à la complète élaboration de la problématique des rapports forme/substance, achèvement qui spécifie en regard la glossématique hjelmslevienne. Il faut déjà noter, à cet égard, la contradiction constitutive de la perspective martinettienne - donc de la critique martinettienne de Hjelmslev -, qui consiste à affirmer la nécessité de prendre en compte la substance, et ainsi à promouvoir l'édification d'une structure dès lors conçue comme substantielle, et non purement formelle, tout en opposant forme et substance, opposition conçue comme un cadre commun aux différentes théories structuralistes. Cette contradiction apparaît de manière très nette dans « Some Basic Principles of Functional Linguistics » (1976), où Martinet affirme d'une part que, face au "rejet [glossématique] de toutes les substances au profit des relations pures » (Martinet, 1977 : p. 11 [nous traduisons]), dans le cadre de la linguistique fonctionnelle, « [l]a substance pertinente est retenue afin de qualifier les relations » (Martinet, 1977 : p. 11 [nous traduisons]), opposant ainsi des « relations pures » à des relations « qualifiées » par la substance, mais également, d’autre part, que tandis que les glossématiciens manifestent une « indifférence à l'égard des changements affectant la substance » (Martinet, 1977 : p. 11 [nous traduisons]), « [l]es changements dans la substance sont conçus [par les fonctionnalistes] comme pavant la voie à un remaniement du réseau relationnel » (Martinet, 1977 : p. 11 [nous traduisons]), où s'opposent cette fois nettement forme ou structure d'une part, et substance d'autre part $^{7}$.

\title{
3 Une structure tout à la fois formelle et substantielle
}

L'opposition forme/substance prend chez Martinet deux formes, que nous envisagerons successivement : celle d'une opposition forme/substance modelée, dans la logique de la lecture martinettienne de 
Hjelmslev $^{8}$, par l'opposition syntagmatique/paradigmatique, et celle de la distinction fonction/substance qui fonde la phonologie martinettienne, et avec elle la linguistique fonctionnelle.

\title{
3.1 Forme/substance et syntagmatique/paradigmatique
}

Martinet identifie la forme hjelmslevienne aux relations syntagmatiques. On lit ainsi par exemple dans «Phonetics and Linguistic Evolution » (1957) : « Mais, à cause de son insistance sur la priorité absolue des relations syntagmatiques ("forme") et de sa complète négligence de fait de la substance phonique et sémantique, la glossématique s’est privée de toute prise sur les données évolutives. » (Martinet, 1996 : p. 13 [nous traduisons]), et dans le glossaire des Mémoires d’un linguiste (1993) : " "Substance”. Chez Hjelmslev, tout ce qui ne ressortit pas aux conditions strictement formelles de coexistence dans la chaîne, comme les substances "sémantique” ou "phonique”. » (Martinet, 1993 : p. 378). Cette assimilation de la forme aux relations syntagmatiques, et réciproquement, de la substance aux relations paradigmatiques, vaut en réalité plus largement pour la linguistique martinettienne. On retrouve ainsi dans « Phonetics and Linguistic Evolution » l’argument diachronique opposé par Martinet à Hjelmslev en faveur de la prise en compte de la substance, mais formulé en premier lieu en termes de "prise en compte de la réalité paradigmatique $^{9} »$ :

\begin{abstract}
« Le structuralisme a ainsi, une fois pour toutes, placé l'étude des éléments distinctifs (normalement phonétiques) au centre des préoccupations linguistiques, mais ceux qui, aujourd’hui, se proclament structuralistes ne sont pas tous équipés pour s’attaquer aux problèmes de la phonologie historique avec quelque espoir d'aller plus loin que leurs prédécesseurs. Seuls ceux qui n’ont jamais perdu de vue la réalité paradigmatique, c'est-à-dire le fait que les locuteurs ont, à chaque point de la chaîne, à choisir parmi un certain nombre d'unités afin de dire ce qu'ils veulent dire, ont les moyens de reposer utilement ces problèmes. Les linguistes pour qui le système d'une langue se compose exclusivement des lois de distribution, dans la chaîne parlée, des diverses unités, significatives et distinctives, n'ont aucune chance de promouvoir la recherche. Afin de comprendre comment et pourquoi un système est susceptible de changer, il faut concevoir celui-ci comme un complexe d'unités dont la nature substantielle, phonique ou sémantique, est dans une étroite dépendance de celle des unités voisines du même système. » (Martinet, 1996 : p. 13-14 [nous traduisons]).
\end{abstract}

Or, par ailleurs, la structure martinettienne est double, syntagmatique, mais également paradigmatique. On lit ainsi notamment dans « Structural Variation in Language » (1962) :

\begin{abstract}
"L'étude des variations structurales à travers le temps, quelles que soient les restrictions attachées au terme "structural”, ne peut être menée à bien sans que les différences substantielles, phoniques comme sémantiques, qui existent entre les unités des mêmes classes, soient prises en considération. Un genre de structure doit être trouvé entre les unités de chaque classe. Pour des raisons de clarté, ce genre de structure devrait être distingué de la structure résultant des relations existant entre les diverses classes. La première devrait être appelée "structure paradigmatique", la seconde "structure syntagmatique". Toutes deux, étant caractéristiques du langage, ne devraient pas être autre chose que deux aspects de la structure linguistique.» (Martinet, 2000 : p. 238-239 [nous traduisons]).
\end{abstract}

Conformément à l'association entre paradigmatique et substance ${ }^{10}$, le «genre de structure » qui «doit être trouvé entre les unités de chaque classe » est lié aux « différences substantielles, phoniques comme sémantiques, qui existent entre les unités de chaque classe ». Dans le même temps, structure paradigmatique et structure syntagmatique sont « deux aspects de la structure linguistique ». La structure martinettienne semble ainsi substantielle en même temps que formelle ${ }^{11}$. La forme martinettienne est en réalité doublement déterminée : par la structure - la reconnaissance du caractère structuré de tout idiome - et par la problématique des rapports son/sens, double détermination qui apparaît de manière très nette dans «Substance phonique et traits distinctifs » (1957/1965) où, après avoir évoqué la « répugnance » (Martinet, 1965a : p. 132) de certains structuralistes pour la définition des unités phoniques en traits pertinents, structuralistes qui dès lors, «étendent, en principe, aussi loin que possible la définition 
syntagmatique des unités, et, pour le domaine phonique (ou de l'expression), [...] se contentent, en pratique, d'énumérer les phonèmes dans un ordre arbitraire » (Martinet, 1965a : p. 132), comportement « dicté par l'idée, qui n'est pas sans fondement, que l'analyse perdra nécessairement de sa rigueur dans la mesure où elle fera intervenir la substance, phonique ou sémantique » (Martinet, 1965a : p. 132), affirme :

\begin{abstract}
«On objectera à cela que, sur le plan phonique, personne n’a jamais pu jusqu'ici s'abstraire de la substance. Il ne faut pas oublier que le langage est un moyen pour communiquer, à l'aide de quelque chose qui est manifeste, autre chose qui ne l'est pas. Ce quelque chose qui est manifeste est de la substance phonique, et, quoi qu'il fasse, le linguiste devra affronter cette substance et s'habituer à reconnaître quels usages les langues individuelles font de ses modalités. C'est ce que recommande et enseigne la phonologie. Au sens large du terme, la phonologie est une phonétique fonctionnelle et structurale qui, pour chaque état de langue, établit une hiérarchie des faits phoniques fondée sur leur rôle dans le procès de communication. » (Martinet, 1965a : p. 133).
\end{abstract}

Apparaît en effet ici, dans le cadre de l'affirmation de la nécessité de prendre en compte la substance, la définition de la langue comme instrument de communication, et avec elle la distinction fonction/substance qui est constitutive de la linguistique fonctionnelle, et qui apparaît à certains égards comme une transposition, dans le domaine des unités phoniques, de la dualité son/sens définitoire du signe.

\title{
3.2 L'opposition fonction/substance: de la problématique des rapports forme/substance à la problématique des rapports son/sens
}

Comme l'affirme Martinet lui-même dans « Fonction et structure en linguistique » (1971), la linguistique martinettienne est tout entière fondée sur le principe de pertinence :

«L'étude structurale du langage se fonde sur une analyse, opérée selon le principe de
pertinence, qui permet de déterminer ce qui, dans la réalité physique observée, est
proprement linguistique et d'établir un classement et une hiérarchie des faits fondés
sur leur fonction respective dans la langue étudiée. » (Martinet, 1975a : p. 33).

Apparaît ici la notion de fonction, et ce principe détermine de fait une opposition entre fonction et substance. Dans le cadre phonologique, fonction et substance apparaissent tout d'abord comme deux critères de classement des sons. Citons par exemple « La notion de fonction en linguistique » (1971) :
«En fait, bien avant que Hjelmslev élabore et publie sa théorie du langage, les phonologues pragois s'étaient fait les hérauts d'une linguistique fonctionnelle où les faits étaient identifiés et classés, non plus sur la base de leur nature physique, mais sur celle du rôle qu'ils jouent dans la communication langagière. C'est dans ce sens que la phonologie a pu être présentée comme une étude fonctionnelle et structurale où la structure est révélée par l'examen de la fonction des éléments. » (Martinet, 1975a : p. 95-96).

On notera, dans ce passage, l'affirmation selon laquelle «la structure est révélée par l'examen de la fonction des éléments ». Au classement s'ajoute en effet la dimension de l'extraction, qui apparaît par exemple dans l'extrait suivant de « Où en est la phonologie ? » (1949) :

«Dégager les traits pertinents, c'est-à-dire ceux des faits de substance phonique qui assurent la fonction distinctive, fonction fondamentale du langage humain, est précisément le moyen de faire le départ entre ce qui est décisif et le reste. Ce n’est pas le phonème, mais le trait pertinent qui est l'unité de base de la phonologie. C'est ce que nous retenons de la substance, c'est la seule unité pour laquelle nous postulions une existence réelle. » (Martinet, 1965a : p. 75).

Or, dans cette perspective, la structure se confond avec les traits pertinents de l'objet, extraits de la substance. On lit ainsi dans « Structure et langue » (1965) : 


\begin{abstract}
« De même qu'en matière d'architecture il est une fonction fondamentale qu'on peut désigner comme la protection et qui détermine ce qu'on appellera la structure de l'édifice, il y a, en matière de langage, une fonction fondamentale, celle de communication, qui détermine ce qu’on appellera la structure de chacune de ces modalités du langage qui reçoit le nom de langue. Est, en dernière analyse, pertinent dans l'édifice ce qui contribue à assurer la protection ; est pertinent, dans la langue, ce qui participe à l'établissement de la communication. En d'autres termes, la structure, de part et d'autre, se confond avec ce que nous désignons comme les traits pertinents de l'objet. » (Martinet, 1965b : p. 293).
\end{abstract}

C’est là ce que Martinet appelle « réalité fonctionnelle », réalité fonctionnelle qui lui paraît définitoire de la langue. Ainsi que nous nous efforcerons de le montrer dans ce qui suit, cette représentation est néanmoins éminemment problématique, et nous reconduit à la dualité, mise en évidence ci-dessus, de la structure martinettienne, tout à la fois formelle et substantielle.

Dans nombre de textes de Martinet, la distinction fonction/substance apparaît corrélative d'une opposition entre invariant et variations. Il est ainsi question dans «Phonetics and Linguistique Evolution » (1957) d' " unités discrètes, variables chacune pour ce qui concerne leur réalisation dans le discours, mais invariables dans l'accomplissement de leur fonction distinctive » (Martinet, 1996: p. 17-18 [nous traduisons]), et Martinet écrit en 1967 dans « La vie secrète du langage » :

«Ce qui est scandaleux chez le phonème, c'est non seulement qu'il n'est phonème que parce que sans nuances, mais que c'est précisément en sa qualité de fait humain qu'il s'oppose, par son monolithisme fonctionnel, à l’infinie variété de ses réalisations physiques. » (Martinet, 2000 : p. 66).

L’invariant renvoie à la réalité linguistique, que Martinet oppose à la réalité physique et substantielle, comme par exemple ${ }^{12}$ dans ce passage des Éléments de linguistique générale $\left(1960^{13}\right)$ :

\begin{abstract}
« [...] l'identité physique ne permet pas de conclure à l’identité linguistique ; un même phonème se réalise différemment selon l'entourage, et un même son, selon l'entourage, peut être la réalisation de phonèmes différents. " (Martinet, 1980/1991 : p. 68-69).
\end{abstract}

Il renvoie ainsi à un autre de la substance, qui permette d’ordonner linguistiquement celle-ci. Au niveau phonique, cet autre de la substance est constitué par la définition phonologique, opposée à une définition phonétique, et qui fonde l'identité linguistique en tant que distincte de l'identité matérielle. On lit notamment dans les Éléments de linguistique générale :
«Avant donc de procéder à l'établissement de l'inventaire des phonèmes, il faudra définir chaque segment en précisant ce qui, dans son environnement phonique, le distingue de tous ceux qui auraient pu y figurer. Une fois ceci terminé, on identifiera comme des réalisations d'un seul et même phonème les segments provenant de contextes différents qui présentent la même définition. » (Martinet, 1980/1991 : p. 69).

Citons également ce passage de «La phonologie » (1938), où l’opposition des deux types de définition apparaît de manière très nette :
« L'énumération ou, comme on dit, l'inventaire des phonèmes de la langue étudiée, est parfois conçue comme la fin dernière des études phonologiques, alors qu'elle n'en est, en fait, que la base. Ces phonèmes que l'on vient d'isoler, il va s'agir maintenant de donner de chacun d'eux une définition phonologique. Pour cela, on ne retiendra de leurs caractéristiques que celles qui sont pertinentes, c'est-à-dire qui l'opposent aux autres phonèmes à l'exclusion des autres qui ne sauraient présenter d'intérêt que pour le phonéticien. Comme nous l'avons vu, ce qui est pertinent dans une langue peut fort bien ne pas l'être dans une autre. Il en résulte que ce qui est phonétiquement semblable peut fort bien être défini par le phonologue en termes différents, selon le système dont il s'occupe. » (Martinet, 2000 : p. 261).

On voit que la définition phonologique est une définition en termes de traits pertinents, et qu'elle nous reconduit ainsi à la dimension de l'extraction. Or, dans cette perspective, la structure martinettienne est à 
la fois substantielle, dans la mesure où elle se confond avec les traits pertinents de l'objet ${ }^{14}$, et formelle, en tant qu'autre de la substance, définitoire d'une identité proprement linguistique et distincte de la réalité physique ou phonétique. Apparaît en effet également, dans les textes de Martinet, en lien avec la distinction entre fonction et substance, la notion de substance formée. Il est ainsi question dans Économie des changements phonétiques d'un «type d'organisation sui generis qui transcende les ressemblances accidentelles entre les réalisations d'unités isolées » (Martinet, 1955 : p. 63), et on lit par ailleurs dans «Structural Linguistics » $(1953)^{15}$, où l’on relève, en lien avec la notion de pertinence, les expressions de « substance linguistiquement formée » et de « substance [...] amorphe » :

\begin{abstract}
« Le principal objet de la recherche praguoise est la substance linguistiquement organisée. Comme telle, la substance, sur les deux plans, est amorphe. L'identité substantielle n’a aucune validité linguistique : le [k] allemand et le [k] tcherkesse peuvent bien être prononcés de la même manière, mais ils ne peuvent pas être considérés comme étant linguistiquement les mêmes parce que l'un et l'autre, le $/ \mathrm{k} /$ allemand et le $/ \mathrm{k} /$ tcherkesse, ne sont ce qu'ils sont qu'en référence aux autres phonèmes de leur structure respective. Le $/ \mathrm{k} /$ allemand est la somme de tous les traits substantiels qui le distinguent régulièrement des autres phonèmes de la structure allemande, et il en va de même du $/ \mathrm{k} /$ tcherkesse dans le cadre de la structure tcherkesse. Tout trait substantiel réel de l'articulation du /k/ allemand, tel que l'action pulmonaire nécessaire à la production de celui-ci, qui n’est pas utile pour le garder distinct d'un autre phonème allemand, est linguistiquement non-pertinent. Mais tout trait qui exerce une fonction distinctive est une partie de la substance sur laquelle l'attention du linguiste doit être concentrée. Tout ce qui, dans la substance, est considéré comme exerçant une fonction communicative, dans le sens ordinaire du terme, est linguistiquement pertinent. » (Martinet, 1953 : p. 583 [nous traduisons]).
\end{abstract}

Martinet ajoute alors : « Les unités qui se révèlent exercer le même type de fonction sont classées selon la nature substantielle de leurs traits linguistiquement pertinents. La structure résulte des diverses combinaisons de ces traits. » (Martinet, 1953 : p. 583 [nous traduisons]), où l'on retrouve la notion de structure comme réalité fonctionnelle.

Il est remarquable, eu égard à cette dualité de la structure martinettienne, que si l'invariant martinettien, bien que substantiel, fait ainsi figure d'existant «formel », dans la mesure où il impose à la substance dans laquelle il se réalise une identité linguistique abstraite, et si corrélativement, la structure, organisatrice de la substance, se situe du côté de la forme, la distinction forme/substance ne se distingue jamais réellement de la distinction fonction/substance, et avec elle, sens/son. L'oscillation entre les deux distinctions apparaît par exemple dans « La vie secrète du langage » (1967), lorsque l’identité linguistique subsomptrice de différentes réalisations se trouve glosée en termes de valeur, au sens de l'appariement d'un son et d'un sens - ici d'une fonction :
«Derrière l'infinie variété distinctive des productions phoniques de l’homme, ils [les phonologues] découvrent des unités distinctives, les phonèmes, dont chaque langue présente un nombre déterminé. Cela implique que le phonème est une unité "discrète" qui est présente ou absente, sans qu’il puisse être question qu’elle soit jamais, ici ou là, plus ou moins ceci ou cela. Le phonème est linguistiquement toujours identique à lui-même, quelles que soient les différences physiques qu'on pourrait constater d'une de ses réalisations à une autre, chez différentes personnes et dans diverses circonstances. En d'autres termes, le phonème est une valeur, comme un billet de cent francs qui vaut cent francs, non du fait de sa réalité physique à base de cellulose, mais parce qu'un groupe d'êtres humains tombe d'accord pour lui conférer un certain pouvoir dans les échanges. » (M.Vi. : p. 65).

Elle apparaît également à travers l'ambivalence de la notion d'utilisation qui est corrélative de l'opposition entre fonction et substance, et qui implique tout à la fois une instance organisante ou informante - structure ou langue -, dont Martinet insiste sur l'indépendance à l'égard de la réalité physique, et la dimension de l'attribution (d'une fonction à un son). On lit ainsi dans « Le point de vue fonctionnel en grammaire » $(1982)^{16}$ : 


\begin{abstract}
« Les implications du point de vue fonctionnel en phonologie sont assez bien connues et ne nous intéressent pas directement ici. Il est bon toutefois de les rappeler parce qu'elles illustrent bien la façon dont chaque langue utilise à ses propres fins les données anatomiques et physiologiques des organes dits "de la parole”, attribuant arbitrairement, au sens saussurien du terme, telle valeur à tel trait, reléguant tel autre au paralinguistique [...] Une même réalité physique, comme la mélodie du discours, peut assumer, d’une langue à une autre et - dans une même langue - d'un point à un autre du discours, des fonctions diverses, distinctive, contrastive, notificative, voire directement significative. » (Martinet, 1989 : p. 53-54).
\end{abstract}

Martinet affirme de même dans « Function and Segmentation in Prosody » (1972) que « les structures linguistiques manifestent un grand degré de liberté par rapport à la nature physique des traits qu'elles emploient » (Martinet, 1989 : p. 120 [Martinet, 1973 : p. 203]) ${ }^{17}$.

Il apparaît donc que le phonème martinettien peut être caractérisé aussi bien comme un son muni d'une fonction, comme dans La description phonologique (1956), où Martinet affirme que " [1]es phonèmes se révèlent [...] comme des habitudes motrices qui se manifestent dans des conditions et à des fins bien définies » (Martinet, 1956: p. 34), que comme un invariant immanent à la substance, ce pour quoi, précisément, la structure martinettienne est double, formelle en même temps que substantielle. La problématique des rapports forme/substance apparaît ainsi de manière on ne peut plus nette comme un effet de l'absence de théorisation du rapport son/sens ou du «fait linguistique ${ }^{18}$ »: l'élaboration martinettienne ne dit jamais rien d'autre que le rapport de la langue à la substance, celui-ci présupposant la donnée d'un rapport son/sens, d'une part, du caractère structuré constatable en tout idiome d'autre part. C'est ce qui apparaîtra d'une autre manière à l'examen de la construction martinettienne de la langue comme structure orientée du son vers le sens.

\title{
4 Redoublement de la distinction fonction/substance et construction de la langue comme structure orientée : l'insistance du fait linguistique comme problème
}

La structure martinettienne est une structure de structures, dont les différentes structures constitutives sont conçues comme "différents niveaux de fonctionnement » (Martinet, 1977 : p. 10 [nous traduisons]) de la langue, dont l'articulation vise à rendre compte du rapport entre son et sens définitoire de cette dernière comme instrument de communication. Cette représentation de la langue comme structure orientée du son vers le sens est construite grâce à un double redoublement de la distinction fonction/substance, c'est-àdire grâce à une application du principe de pertinence présidant à l'établissement de la réalité fonctionnelle phonologique et de la distinction entre phonétique et phonologie aux autres niveaux de fonctionnement de la langue que le niveau phonique. On lit ainsi dans les Mémoires d'un linguiste (1993), en réponse à une question de Georges Kassai portant sur «axiologie opposée à sémantique, syntaxe opposée à morphologie, phonologie opposée à phonétique » (Martinet, 1993 : p. 325) :

\footnotetext{
« Les oppositions que vous rappelez se fondent sur le principe de pertinence : pour aborder scientifiquement un objet, il faut appliquer une pertinence ou deux pertinences successives qui établissent des plans différents qu'il ne faut pas entremêler si l'on veut comprendre ce qui se passe. Chacun des membres des paires phonologie-phonétique, syntaxe-morphologie, axiologie-sémantique correspond à une pertinence particulière. » (Martinet, 1993 : p. 325).
}

La distinction phonétique/phonologie se double donc de deux autres, morphologie/syntaxe et sémantique/axiologie, qui résultent d'une application analogue du principe de pertinence. Le fait notable est néanmoins qu'en dépit de cette commune origine, il n'existe pas véritablement de parallélisme entre les deux couples morphologie/syntaxe et sémantique/axiologie, autre qu'un commun parallélisme avec le couple phonétique/phonologie, dont la dualité, mise en évidence dans ce qui précède, apparaît ainsi de manière d'autant plus nette. Comme nous le verrons dans ce qui suit, la représentation martinettienne de la langue répond en effet aux deux exigences contradictoires que sont la symétrie du son et du sens relativement à la question des rapports forme/substance - l'isomorphisme hjelmslevien, contre lequel Martinet s'inscrit en faux, 
promouvant pour sa part la notion de double articulation - et la construction d'une structure orientée du son vers le sens, quant à elle fondée sur une sorte de redoublement vertical de la distinction fonction/substance, contradiction où insiste, de nouveau, le problème de la théorisation du rapport son/sens.

La distinction fonction/substance se redouble tout d'abord en distinction sens/forme (au sens de signifiant). On lit ainsi en 1964 dans « The Foundations of a Functional Syntax » :

\begin{abstract}
«Une approche délibérément fonctionnelle de la phonologie implique normalement que l'étiquetage et le groupement des faits soient déterminés par le rôle que jouent ces faits dans le procès de communication plutôt que par leur nature physique [...]

De la même manière, une approche délibérément fonctionnelle de la syntaxe impliquera que les ressemblances formelles soient subordonnées à l'identité fonctionnelle. [...]

[...] Le point de vue fonctionnel nous rend ainsi indépendants des caprices des formes linguistiques. » (Martinet, 1975a : p. 118-119 [nous traduisons]).
\end{abstract}

Martinet établit ici entre sens (ou fonction) et forme (au sens de signifiant) une hiérarchie qui rappelle le rapport établi entre fonction et substance, fonction et sens étant érigés au statut de critère pour l'ordonnance de la substance ou des formes. De la même manière que la fonction permettait d'identifier les unités phonologiques, le sens (ou la fonction significative) permet l'identification des unités significatives, c'est-à-dire, dans la terminologie de Martinet, des monèmes, unités significatives minimales. Citons par exemple « Des limites de la morphologie » (1965) :
«Comme on le voit, le contexte qui détermine le choix d’une forme du signifiant ou de l'autre peut être un contexte phonique (consonne ou voyelle suivante) ou un contexte grammatical ( $3^{\mathrm{e}}$ personne du singulier ou $1^{\mathrm{re}}$ personne du pluriel), mais, dans l'un et l'autre cas, l'identité du monème n'est pas affectée par le choix d'une succession phonématique différente : beau et bel ont exactement le même sens ; /l/ et /y/ sont identifiables l'un et l'autre comme l'article défini masculin ; /va/ et /al-/ ont, en tout, la même valeur. » (Martinet, 2000 : p. 380-381).

Comme l'affirme Martinet dans ses Mémoires d'un linguiste, «l'identité du signe minimal n’est pas un problème de forme, mais un problème de signifié » (Martinet, 1993 : p. 347). On retrouve d'ailleurs, modelant cette distinction sens/forme, le couple invariant/variation, le monème étant par exemple défini dans « Autour du syllemme » (1980) comme « une nouvelle version du signe minimum identifié sur la base de son signifié et sans considération des variantes de son signifiant » (Martinet, 1989 : p. 136), et dans les Éléments de linguistique générale (1960), la morphologie comme « [1]'étude des variantes de signifiant » (Martinet, 1980/1991 : p. 106). Par ailleurs, tout comme sur le plan phonique la fonction est le critère d'une réalité phonologique différente de la réalité physique, au niveau des unités significatives, le sens est le critère d'une réalité fonctionnelle, de même opposée à une réalité physique. Martinet écrit ainsi en 1962 dans « De la variété des unités significatives » ${ }^{19}:$ « Il y a une réalité fonctionnelle du langage qui doit être cherchée au-delà d’accidents purement formels » (Martinet, 1965a : p. 184).

Le parallélisme est cependant loin d'être complet entre les deux distinctions fonction/substance et sens/forme. En premier lieu, tandis que sur le plan des unités phoniques l'identification des unités est tout entière subordonnée à la fonction, sur celui des unités significatives, en dépit de la subordination de la forme au sens, le signifiant a un rôle à jouer dans l'identification du monème, conformément à la logique même de la structure orientée, dont le soubassement est un système de phonèmes à fonction distinctive. On lit ainsi dans Syntaxe générale (1985) :

\begin{abstract}
«Pour prendre conscience de la spécificité de la structure d’une langue particulière, il convient donc de dégager en priorité les unités distinctives de base, les phonèmes. Ceux-ci permettant de faire correspondre, à chaque signifié, une forme perceptible assurant son identité, il ne reste plus qu’à préciser comment les signes peuvent se combiner pour communiquer l'expérience et à quel aspect de l'expérience correspond chacun d'eux. Une fois établi le système des unités distinctives assurant l'identité du signe, on doit donc pouvoir passer directement à ce que, de façon lâche, on désigne comme la syntaxe et la sémantique. » (Martinet, 1985 : p. 10).
\end{abstract}


Martinet insiste ainsi à quelques reprises sur la spécificité des variantes de signifiant, au regard des variantes phoniques, comme dans les Éléments de linguistique générale ${ }^{20}$, où il affirme qu' « il y a, entre les variantes des signifiants et les variantes des phonèmes, une différence fondamentale qui tient au fait que les variantes de phonème ne se définissent pas en termes de grandeurs discrètes : toute réalisation d'un phonème est une variante puisque, physiquement, elle diffère, tant soit peu, de toute autre, du fait du contexte ou de l'humeur du locuteur » (Martinet, 1980/1991: p. 107), tandis que «les variantes de signifiant se définissent en termes de phonèmes, c'est-à-dire d'unités discrètes » (Martinet, 1980/1991 : p. 107), de sorte qu' «[u]n signifiant ou une variante de signifiant est toujours identifiable en termes d'unités distinctives discrètes ou de zéro » (Martinet, 1980/1991 : p. 107). De fait, tandis que les rapports entre fonction et substance sont d'organisation, et non seulement d'appariement, les rapports du sens à la forme sont de simple évaluation, et la dimension de l'extraction ne vaut pas pour cette distinction : la sélection des formes pertinentes se fait par rattachement à un (non-pertinence) ou plusieurs (pertinence) signifiés, là où en ce qui concerne la substance phonique, le classement des variantes était corrélatif de l'extraction et d'une définition constitutive d'un invariant dès lors tout à la fois substantiel et formel. Il n’y a donc pas, à ce premier niveau des unités significatives, d'existant formel, celui-ci cédant la place au signifié, distinct, en tant que tel, du signifiant dont il permet l'analyse. Il apparaît ainsi qu'alors que la problématique martinettienne est fondamentalement une problématique des rapports son/sens, la dimension de la forme n'émerge de cette dernière que dans la mesure où il s'agit de substance, et d'une attribution ou d'une analyse qui est ainsi intrinsèquement organisation.

La distinction sémantique/axiologie implique quant à elle en premier lieu les notions de structure et d'intégration. On lit ainsi dans « Fonction et pertinence communicative » (1980) :

\begin{abstract}
« Nous arrivons maintenant au problème du sens. Et là je pense qu'il faut distinguer entre deux disciplines. De même que nous distinguons entre la phonétique et la phonologie, il nous faut distinguer entre la "sémantique" et autre chose. La phonologie est l'étude des unités distinctives qui s’opposent. Sur le plan de la signification, il nous faut une discipline qui oppose les valeurs résultant des oppositions. À partir du grec axia “valeur” j'ai fait axiologie. L'axiologie est donc l'étude des valeurs signifiées qui s’opposent. » (Martinet, 2000 : p. 121).
\end{abstract}

Aussi s’agit-il moins alors de redoublement de la distinction fonction/substance que d'opérativité de la distinction forme/substance, comme il apparaît de manière très nette à la lecture du texte qui marque l'acte de naissance de la distinction sémantique/axiologie, «Arbitraire linguistique et double articulation » (1957). Martinet note en effet dans ce texte qu' «il n’y a [...] aucune discipline paralinguistique qui corresponde à la "phonétique" (par opposition à la "phonologie”) et qui nous permette de traiter d'une réalité psychique antérieure à toute intégration aux cadres linguistiques » (Martinet, 1965a : p. 30), et que «même en matière d'examen de la réalité psychique intégrée à la structure linguistique, on n'a rien [...] qui soit le pendant de ce qu'est la phonologie sur le plan des sons » (Martinet, 1965a : p. 30), ce qu'il illustre par le schéma suivant ${ }^{21}$ :

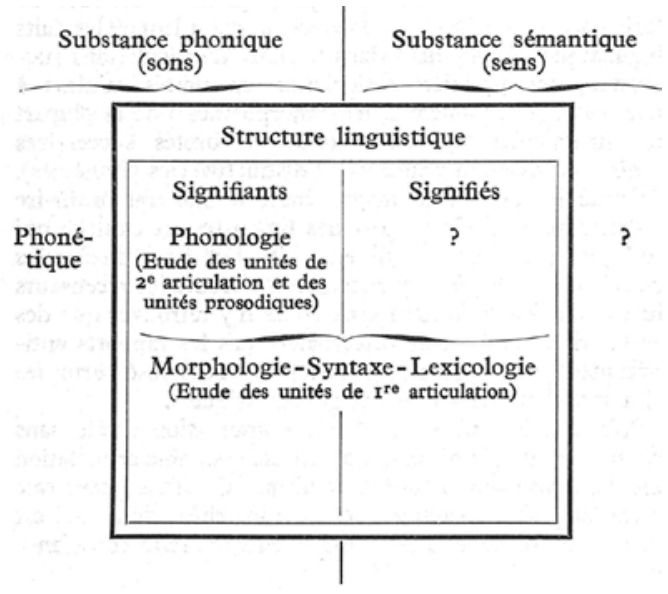


Or, ce schéma est remarquable, en ce que s’y conjuguent deux schèmes, celui de la double articulation, qui le structure verticalement, et celui qui oppose substance et structure, qui divise la première tranche horizontale en quatre parties comparables, mutatis mutandis, à la quadripartition glossématique forme/substance/du contenu/de l'expression, de sorte que l'opposition phonétique/phonologie et la future opposition sémantique/axiologie y apparaissent comme étant totalement déterminées par la dichotomie forme/substance. Il sera de même question par exemple dans « Pour une linguistique des langues » (1973) de la phonétique et de la sémantique comme des "domaines de l'expression et du contenu non formés » (Martinet, 2000 : p. 26), Martinet ajoutant, à propos de la sémantique, qu'elle « correspondrait à l'expérience humaine antérieurement à son modelage par l'exercice d'une langue » (Martinet, 2000 : p. 27). La distinction entre sémantique et axiologie est donc avant tout une distinction entre forme et substance, parallèle, en cela, à la distinction entre phonétique et phonologie, mais significativement différente de celle entre morphologie et syntaxe. Si le couple invariant/variations vaut également pour l'opposition axiologie/sémantique ${ }^{22}$, Martinet insiste d'ailleurs inversement sur l'analogie existant entre les variantes phoniques et significatives. On lisait ainsi dans les Éléments de linguistique générale, en suite du passage cité ci-dessus ${ }^{23}$ :

« Il en va tout autrement de la face signifiée du monème, dont la valeur varie selon les contextes ou les situations aussi largement que la réalisation d'un phonème : que l'on compare il court après l'autobus, il court après la fortune, il court le cerf, c'est un coureur (prononcé dans un stade ou dans un salon). » (Martinet, 1980/1991 : p. 107).

Le parallélisme n'est cependant pas complet entre les deux distinctions phonétique/phonologie et sémantique/axiologie. En effet, tandis qu'il y a sur le plan phonique une étroite corrélation entre fonction et structure, les deux aspects ${ }^{24}$ demeurent relativement indépendants sur le plan sémantique. En témoigne par exemple, cet extrait de « Sémantique et axiologie » (1975) :

\begin{abstract}
"Lorsqu'on travaille sur la substance phonique, on dispose des deux termes de "phonétique” et de "phonologie”, l’un pour désigner l'étude de la réalité phonique de la parole en elle-même et indépendamment de son intégration à une structure linguistique particulière, l'autre pour désigner l'examen de la hiérarchie fonctionnelle des traits phoniques dans une langue donnée. Sur le plan de la substance sémantique, on n'avait aucune possibilité de distinguer entre un traitement de la réalité psychique en elle-même et indépendamment de son intégration à une structure déterminée et l'étude de cette réalité organisée dans le cadre d’une langue particulière. » (Martinet, 1975 b : p. 539).
\end{abstract}

L’apparente régularité de structure de ce passage recèle en effet une légère asymétrie, puisqu'au couple « étude de la réalité phonique de la parole en elle-même et indépendamment de son intégration à une structure linguistique particulière »/« examen de la hiérarchie fonctionnelle des traits phoniques dans une langue donnée » répond une opposition uniquement centrée sur la structure : "traitement de la réalité psychique en elle-même et indépendamment de son intégration à une structure déterminée »/ «étude de cette réalité organisée dans le cadre d'une langue particulière ». On lit de même dans « La présentation des unités significatives » (1976) :
«On peut dire que l'axiologie est à la sémantique ce que la phonologie est à la phonétique et, de même qu'on peut parler d'une phonétique distinctive où l'on traite de différences phoniques qui peuvent servir à des fins phonologiques, on pourra parler de sémantique axiologique lorsqu'on traite, indépendamment de toute langue existante, de distinctions de sens susceptibles d'entrer dans un système de valeurs. » (Martinet, 1976 : p. 152).

Cette asymétrie entre phonologie et axiologie semble inhérente à la notion de structure orientée, qui implique, en tant que telle, un mouvement du son vers le sens. Or, il faut noter, à cet égard, que l'analyse permettant de construire cette structure implique pour sa part un double mouvement du son vers le sens et du sens vers le son, et dès lors un retournement, en soi problématique. Ce retournement est lisible dans le double parallélisme qui préside à la construction de la hiérarchie structurale et fonctionnelle : la distinction morphologie/syntaxe est parallèle à la distinction phonétique/phonologie en tant que produit d'un redoublement de la distinction substance/fonction ; la distinction sémantique/axiologie est parallèle à 
la distinction phonétique/phonologie en tant que nouvelle distinction substance/forme ; se trouve ainsi disjoint ce que la distinction phonétique/phonologie peut conjoindre dans la mesure où elle ne distingue pas entre son/sens et forme/substance, et dès lors à la jonction du son et du sens se produit un retournement qui inverse le rapport du signifiant au signifié : de délimitant, le signifié devient délimité. De fait, si, en ce qui concerne le couple morphologie/syntaxe, le rôle de subsompteur de variantes revenait au signifié, il revient, en ce qui concerne le couple sémantique/axiologie, au signifiant. On lit notamment dans les Mémoires d'un linguiste (1993) :

\begin{abstract}
« Prenons le cas de la sémantique opposée à l’axiologie. À un point de la recherche, on écarte tous les faits de sens qui ne correspondent pas à une différence de forme. On dégage donc, en français, un "passé composé” comme une unité minimale s’opposant à l’imparfait, au futur, etc. Cela fait, on peut relever que selon le verbe et le contexte, le "passé composé" est un accompli (j’ai fini!) ou un passé (j’ai fini hier à cinq heures). Et là, nous sortons de l'axiologie pour trouver la sémantique. » (Martinet, 1993 : p. 325).
\end{abstract}

Le recours au signifiant comme garant de l'unicité du signifié est un point de parallélisme avec la distinction phonétique/phonologie : de la même manière que la fonction (orientée, en tant que telle, vers le pôle du sens) permet la reconnaissance, sur le plan phonique, d'un invariant subsumant les variations, c’est la forme (le signifiant, orienté, quant à lui, vers la langue) qui en permet la reconnaissance sur le plan sémantique. Le fait n’en pose pas moins problème. Comment, en effet, peut-on sans contradiction poser d'un côté, pour la morphologie, la pertinence du seul signifié pour la délimitation des monèmes, et postuler de l'autre, pour la distinction axiologie/sémantique, que l'unicité du signifiant est le garant de l'unicité du signifié. Pour Martinet, phonologie, morphologie, syntaxe et axiologie correspondent certes à des étapes successives dans la description d'une langue. C’est là, néanmoins, une résolution du problème en termes d'analyse et de description, tandis que Martinet a toujours cherché à élaborer une représentation du fonctionnement de la langue. Ce retournement problématique qui marque la construction martinettienne de la langue comme structure orientée du son vers le sens nous paraît donc un point d'insistance de la non-théorisation du rapport son/sens qui sépare radicalement le structuralisme de la problématique saussurienne, et dont est par ailleurs symptomatique le fonctionnement à la fois corrélatif et désordonné des deux problématiques des rapports son/sens et des rapports forme/substance que nous nous sommes efforcée de faire apparaître dans ce qui précède.

\title{
5 Conclusion
}

L'analyse de la distinction martinettienne entre forme et substance fait ainsi apparaître, en lieu et place d'une représentation cohérente du fonctionnement de la langue, le fonctionnement désordonné de deux problématiques qu'il faut qualifier d'analytiques, dans la mesure où elles ne permettent qu'une construction (au sens objectal, et non bachelardien du terme) de la langue à partir des idiomes, au moyen de l'analyse du donné linguistique, analyse fondée pour sa part sur une définition de la langue demeurant non interrogée dans son évidence : comme instrument de communication et comme structure, et ainsi dénuée de tout enjeu véritablement étiologique. L’élaboration martinettienne se distingue ainsi radicalement de l'élaboration saussurienne, mais également, notamment, de l'élaboration hjelmslevienne, qui fait à cet égard figure de répondant objectal de l'élaboration saussurienne par sa mise en place d'une notion homogène de forme, qui tranche sur la dualité de la distinction martinettienne entre forme et substance, dualité que l'on retrouve, bien que sous une forme un peu différente, chez Jakobson, l'élaboration benvenistienne se caractérisant pour sa part par un fonctionnement minimal de la distinction forme/substance. Apparaissent ainsi, à l'analyse récurrente de ces différentes configurations du paradigme structuraliste, différents obstacles épistémologiques, ou différentes manifestations de ces derniers, dont il nous semble important d'analyser le fonctionnement et les enjeux, dans la mesure où cette analyse est susceptible de rendre le linguiste toujours plus conscient de « ce qu'il fait ${ }^{25}$ » (Saussure, 1964 : p. 95), et par là de contribuer au progrès de la linguistique. On pourra ainsi s'interroger, au terme de cette analyse de l'élaboration martinettienne, sur l’objet visé par la phonologie et par la sémantique : s'il ne s'agit pas de langue, de quelle manière s’agit-il d’idiome, et que représente-t-on ainsi ? 


\section{Références bibliographiques}

Bachelard, G. (1938 [2004]). La formation de l'esprit scientifique. Contribution à une psychanalyse de la connaissance. Paris : Vrin.

Jakobson, R. (1963 [2003]). Essais de linguistique générale, I. Les fondations du langage. Paris : Éditions de Minuit.

Jakobson, R. $\left(1971^{26}\right)$. Selected Writings I. The Hague, Paris : Mouton Publishers.

Jakobson, R. (1973 [1979]). Essais de linguistique générale, II. Rapports internes et externes du langage. Paris : Éditions de Minuit.

Jakobson, R. (1988). Selected Writings VIII. Berlin, New York, Amsterdam : Mouton Publishers.

Jakobson, R. \& Pomorska K. (1980). Dialogues. Paris : Éditions de Minuit.

Jakobson, R. \& Waugh L. (1980). La charpente phonique du langage. Paris : Éditions de Minuit.

Martinet, A. (1953). « Structural Linguistics ». Anthropology Today, Kroeber, A. L. (éd.), p. 574-586. Chicago : The University of Chicago Press.

Martinet, A. (1955 [1964]). Économie des changements phonétiques. Traité de phonologie diachronique. Berne : A. Francke S.A.

Martinet, A. (1956). La description phonologique avec application au parler franco-provençal d'Hauteville. Genève : Droz/Paris : M. J. Minard.

Martinet, A. (1965a [1970]). La Linguistique synchronique. Paris : Puf.

Martinet, A. (1965b). « Structure et langue ». Revue internationale de philosophie, XIX ${ }^{\mathrm{e}}$ année, fasc. 3-4, p. 291-299.

Martinet, A. (1962). A Functional View of Language. Oxford : Clarendon Press.

Martinet, A. (1969). Langue et fonction. Une théorie fonctionnelle du langage. Paris : Gonthier.

Martinet, A. (1972 [1974]). «Saussure (Ferdinand de) 1857-1913», in Encyclopcedia Universalis, vol. XIV, p. 695696. Paris : Encyclopædia Universalis France.

Martinet, A. (1973). « Function and Segmentation in Prosody ». Pàkha Sanjam, vol. vI, p. 202-208.

Martinet, A. (1975a). Studies in Functional Syntax. Études de syntaxe fonctionnelle. Munich : Wilhelm Fink Verlag.

Martinet, A. (1975b). « Sémantique et axiologie ». Revue Roumaine de linguistique, tome Xx, nº 5, p. 539-542.

Martinet, A. (1976). « La présentation des unités significatives ». Revista de letras, vol. XVIII, p. 143-153.

Martinet, A. (1977). « Some Basic Principles of Functional Linguistics ». La Linguistique, vol. XIII, fasc. 1, p. 7-14.

Martinet, A. (1980/1991 [1996]). Éléments de linguistique générale. Paris : Armand Colin.

Martinet, A. (1985). Syntaxe générale. Paris : Armand Colin.

Martinet, A. (1989). Fonction et dynamique des langues. Paris : Armand Colin.

Martinet, A. (1993). Mémoires d'un linguiste. Vivre les langues. Paris : Quai Voltaire.

Martinet, A. (1996). The Internal Conditioning of Phonological Systems. Thiruvananthapuram : International School of Dravidian Linguistics.

Martinet, A. (2000). Les introuvables d'André Martinet (La Linguistique, vol. XXXVI, fasc. 1 et 2). Paris : Puf.

Martinet, A. (dir.) (1979). Grammaire fonctionnelle du français. Paris : Crédif/Didier.

Saussure, F. (de) (1964). "Lettres de Ferdinand de Saussure à Antoine Meillet publiées par Emile Benveniste ». Cahiers Ferdinand de Saussure, $n^{0} 21$, p. 89-130.

Saussure, F. (de) (1967/1974). Cours de linguistique générale, édition critique de Rudolf Engler. Wiesbaden : Otto Harrassowitz.

Saussure, F. (de) (1972 ${ }^{27}$ [1995]). Cours de linguistique générale, édition critique de Tullio de Mauro. Paris : Payot. 
Saussure, F. (de) (1997). Deuxième Cours de linguistique générale (1908-1909), d'après les cahiers d'Albert Riedlinger et Charles Patois. Oxford, New York, Tokyo : Pergamon.

Saussure, F. (de) (2002). Écrits de linguistique générale. Paris : Gallimard ${ }^{28}$.

Saussure, F. (de) \& Constantin, E. (2005). « Ferdinand de Saussure : Notes préparatoires pour le cours de linguistique générale 1910-1911, Emile Constantin : Linguistique générale. Cours de M. le professeur de Saussure 19101911 ». Cahiers Ferdinand de Saussure, nº 58, p. 83-289.

Toutain, A.-G. (2014). La rupture saussurienne. L'espace du langage. Louvain-la-Neuve : Academia-Bruylant.

Toutain, A.-G. (2015). La problématique phonologique. Du structuralisme linguistique comme idéologie scientifique. Paris : Classiques Garnier.

\footnotetext{
${ }^{1}$ Voir Bachelard (1938).

2 « Au sujet des fondements de la théorie linguistique de Louis Hjelmslev », republié in Martinet (2000) : p. 71-94. Nous citons les textes de Martinet dans leur dernière version publiée, et en français, soit qu'une traduction en ait été publiée, soit que nous traduisions. Nous donnerons cependant chaque fois le titre original, et préciserons entre parenthèses la date de composition ou de première publication, dans la mesure où la prise en considération de celle-ci est nécessaire à toute étude épistémologique.

${ }^{3}$ Il en va de même de celle de Jakobson. Voir à cet égard « Phonology and Phonetics », in Jakobson (1971) : p. 115117 [Jakobson (1963) : p. 474-475], le «Retrospect " des Selected Writings I, in Jakobson (1973) : p. 142-143 [Jakobson (1963) : p. 639-640], The Sound Shape of Language (Jakobson \& Waugh, 1980) : p. 58 et 65-69 [Jakobson (1988) : p. 47-48 et 53-57], Dialogues (Jakobson \& Pomorska, 1980) : p. 39-40.
}

${ }^{4}$ À cet égard, il n'est rien de plus instructif que la relecture martinettienne de propositions saussuriennes du Cours de linguistique générale, qui montre que, significativement, Martinet ne distingue pas, sur la question de la dualité forme/substance, entre Hjelmslev et Saussure. Voir notamment « Saussure (Ferdinand de) » (Martinet, 1972) : p. 696, " La pertinence » (1973), in Martinet (2000) : p. 98 et 100 et « La notion de fonction en linguistique », in Martinet (1975a) : p. 97-98.

${ }^{5}$ Voir également, notamment, Saussure (2002) : p. 87-88.

${ }^{6}$ On lit en effet en conclusion du passage : « Le terrain de la linguistique est le terrain commun <qu'on pourrait appeler dans un sens très large le terrain> des articulations, c'est-à-dire des "articuli”, des petits membres dans lesquels la pensée prend conscience <(valeur ? B.) > par un son. » (Saussure, 1997 : p. 22).

${ }^{7}$ On lisait de même dans « Au sujet des fondements de la théorie linguistique de Louis Hjelmslev » : « L'expression est un moyen, le contenu une fin, et ceci aussi bien dans le domaine étroitement linguistique de la forme, que dans celui de la substance où un domaine phonique fort restreint est utilisé pour exprimer tout ce qui est exprimable. » (Martinet, 2000 : p. 92 [nous soulignons]), et, comme dans "Some Basic Principles of Functional Linguistics »: «Or, s'il se révèle que c'est dans la substance, plus que dans la forme, que se trouvent les germes de l'évolution linguistique, l'établissement des structures “algébriques” des glossématiciens nous aura fort mal préparé à l'examen indispensable de la réalité diachronique. » (Martinet, 2000 : p. 89). Voir en outre notamment, pour des formulations analogues de cet argument diachronique, «Structural Variation in Language » (1962), in Martinet (2000) : p. 237238, Mémoires d'un linguiste (Martinet, 1993) : p. 94 et The Internal Conditioning of Phonological Systems (Martinet, 1996) : p. 2.

${ }^{8}$ Par ailleurs contestable.

${ }^{9}$ Voir également à cet égard « Function, Structure and Sound Change » (1952), in Martinet (1996) : p. 40.

${ }^{10}$ Réaffirmée dans le développement mentionné dans la note suivante, ainsi que quelques pages plus loin (voir Martinet, 2000 : p. 242).

${ }^{11}$ Voir également notamment à cet égard, dans le même texte, Martinet (2000) : p. 234-236, ainsi que « Structural Linguistics » (Martinet, 1953) : p. 583-584.

${ }^{12}$ Voir également, notamment, A Functional View of Language (Fonction et langue ; respectivement, Martinet, 1962 et Martinet, 1969) : p. 17-18 [p. 8]. 
${ }^{13}$ Nous citons la version de 1991, mais ce passage est demeuré inchangé depuis la première publication de l'ouvrage en 1960. Il en ira de même des autres citations de cet ouvrage.

${ }^{14}$ Martinet affirme d'ailleurs parfois l'existence d'un substrat neuro-musculaire pour cet invariant. Voir par exemple Économie des changements phonétiques (Martinet, 1955) : p. 178, Martinet (1969) : p. 17-18 et 36-37 [Martinet (1962) : p. 8 et 25], Martinet (1965b) : p. 299, et « Les choix du locuteur » (1966), in Martinet (2000) : p. 324.

${ }^{15}$ Mais voir également, notamment, La description phonologique (Martinet, 1956) : p. 35-36, et « Occlusives and Affricates with Reference to some Problems of Romance Phonology » (1949), in Martinet (1996) : p. 101-102.

${ }^{16}$ Mais voir également Martinet (1965a) : p. 133, cité ci-dessus, ainsi que Martinet (1956) : p. 16 et 82.

${ }^{17}$ Concernant l'indépendance de la fonction à l'égard de la réalité physique, voir notamment, outre Martinet (1989) : p. 119-121, que nous citons ici très partiellement, Martinet (1980/1991) : p. 63 et 88, Martinet (1969) : p. 44-45 [Martinet (1962) : p. 32] et La linguistique synchronique (Martinet, 1965a) : p. 46.

${ }^{18}$ Ce syntagme figure dans l'autre passage-source du paragraphe «La langue comme pensée organisée dans la matière phonique » du Cours de linguistique générale, qui se trouve dans le troisième cours : " Mais il n’y a rien du tout de distinct dans la pensée avant le signe linguistique. Ceci est le principal. D’un autre côté, il vaut aussi la peine de se demander si en face de ce royaume des idées tout à fait confus le royaume du son offrirait d'avance des unités bien distinctes (pris en lui-même en-dehors de l'idée).

Il n’y a pas non plus dans le son des unités bien distinctes, circonscrites d'avance. C’est entre deux que le fait linguistique se passe. [...]

Ce fait < linguistique > donnera naissance à des valeurs qui elles < pour la première fois > seront déterminées, mais qui n'en resteront pas moins des valeurs, avec le sens qu'on peut attacher à ce mot. » (Saussure \& Constantin, 2005 : p. 285).

${ }^{19}$ Voir également, notamment, « Composition, dérivation et monème » (1968), in Martinet (1975a) : p. 176-177.

${ }^{20}$ Voir également Martinet (1985) : p. 47 et « Neutralisation et syncrétisme » (1968), in Martinet (1975a) : p. 75.

${ }^{21}$ Voir Martinet (1965a) : p. 30.

${ }^{22}$ Du moins à partir de 1980. Voir Martinet (2000) : p. 121-122 et 128-131, ainsi que Martinet (1993) : p. 325.

${ }^{23}$ Voir également la Grammaire fonctionnelle du français (Martinet, 1979 (dir.)) : p. 8-9.

${ }^{24}$ L'aspect fonctionnel n'est de fait pas absent de l'axiologie, pour laquelle Martinet parle de même de traits pertinents (voir par exemple Martinet, 2000 : p. 41, et «L'axiologie, étude des valeurs signifiées » (1977), in Martinet, 2000 : p. 439), voire d'utilisation (voir Martinet, 2000 : p. 241).

${ }^{25}$ L'expression figure dans la lettre de Saussure à Meillet du 4 janvier 1894 : « Mais je suis bien dégoûté de tout cela, et de la difficulté qu'il y a en général à écrire seulement dix lignes ayant le sens commun en matière de faits de langage. Préoccupé surtout depuis longtemps de la classification logique de ces faits, de la classification des points de vue sous lesquels nous les traitons, je vois de plus en plus à la fois l'immensité du travail qu'il faudrait pour montrer au linguiste ce qu'il fait; en réduisant chaque opération à sa catégorie prévue ; et en même temps l'assez grande vanité de tout ce qu’on peut faire finalement en linguistique. » (Saussure, 1964 : p. 95).

${ }^{26}$ Il s'agit de l'édition augmentée d'addenda. La première édition des Selected Writings I date de 1962.

${ }^{27}$ La première édition du Cours de linguistique générale date de 1916. Les suivantes (la seconde édition date de 1922) s'en distinguent par quelques modifications mineures et une pagination différente. 1972 est la date de la première édition française - l'édition italienne datant de 1967 - qui soit accompagnée de l'apparat critique de Tullio de Mauro.

${ }^{28}$ Toutes nos citations ont été revues à la lumière des manuscrits. Pour ne pas alourdir ce texte, nous n’indiquons pas les modifications apportées. 